\title{
Quantifying Uncertainty in Ecotoxicological
}

\author{
Risk Assessment: MUST, a Modular \\ Uncertainty Scoring Tool
}

\author{
Jakub Kostal ${ }^{*}+{ }^{+}$, Hans Plugge ${ }^{+}$and Will Raderman ${ }^{+}$ \\ 'Department of Chemistry, The George Washington University, $80022^{\text {nd }}$ ST NW Suite \\ 4000, Washington DC, 20052-0066 \\ ${ }^{\ddagger}$ Safer Chemicals Analytics, Verisk 3E, 4520 East West Highway, Suite 440, Bethesda, \\ MD 20814 USA
}

\section{Supporting Material}

S2 Figure S1. Increase in relative reliabilities (left y-axis) across 3 arbitrary values 1,2 and 3 (x-axis) and corresponding computed uncertainties (right $\mathrm{y}$-axis). Left to right, top to bottom: $R_{f}=\mathrm{x} / 10^{\mathrm{n}}+0.1$ where $\mathrm{n}=4,3,2$ and $1 ; N=3, \sigma_{g}=4$.

S3-S6 Table S1. Acute aquatic toxicity $\left(\mathrm{LC}_{50}\right)$ values extracted from the ECHA database for nickel sulfide (CAS 16812-54-7) with corresponding Klimisch scores (KS), assigned reliability factors $\left(R_{f}=1,0.7,0.3\right.$ and 0.1 for $\mathrm{KS}=1$, 2,3 and 4 , respectively).

S7 Table S2. Acute aquatic toxicity $\left(\mathrm{LC}_{50}\right)$ values obtained using Rainbow trout (Oncorhynchus mykiss) model from the ECHA database for nickel sulfide (CAS 16812-54-7).

S8-S9 Table S3. Acute aquatic toxicity $\left(\mathrm{LC}_{50}\right)$ values extracted from the ECHA database for ethylbenzene (CAS 100-41-4).

S10 Table S4. A questionnaire for the MUST parametrization. 

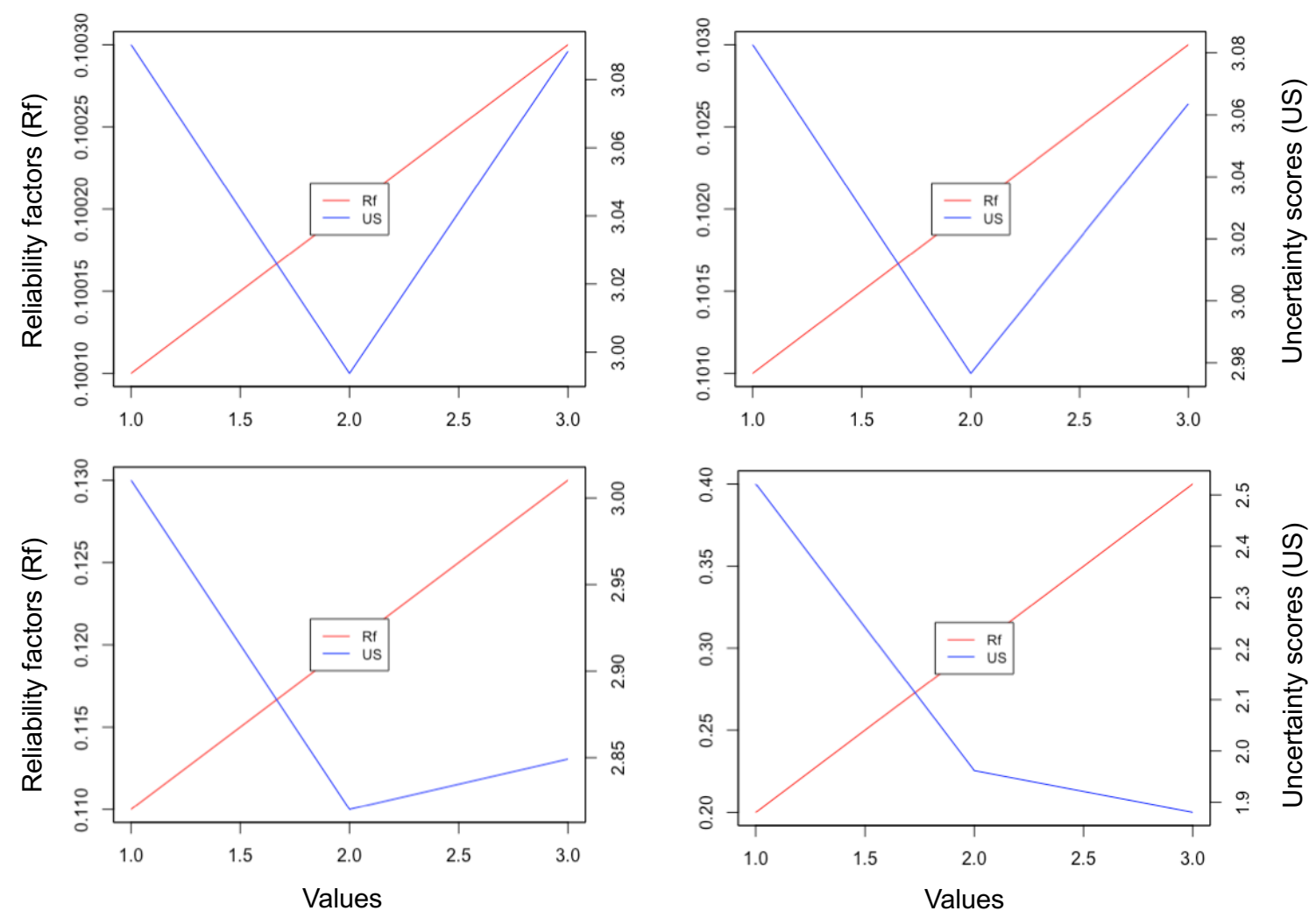

Figure S1. Increase in relative reliabilities (left y-axis) across 3 arbitrary values 1, 2 and 3 (x-axis) and corresponding computed uncertainties (right y-axis). Left to right, top to bottom: $R_{f}=\mathrm{x} / 10^{\mathrm{n}}+0.1$ where $\mathrm{n}=4,3,2$ and $1 ; N=3, \sigma_{g}=4$. As the gradient in relative reliabilities increases (viz. increase in left y-axis scale), preference for the middle value (2) decreases, and eventually value with the highest reliability (3) is favored by computed uncertainty scores. Note: In contrast to Figure $4 b$, middle value (2) is favored even at a gradient of 0.01, whereas in Figure $4 b$ this gradient corresponds to preference for the most-reliable data (3). This 'discrepancy' is due to larger absolute $R_{f}$ values considered here than in Figure $4 b$, which lead to preference for the middle value. This is consistent with our assessment that, ceteris paribus, Equation 1 favors average values over the mostreliable values when absolute reliabilities are large. 
Table S1. Acute aquatic toxicity $\left(\mathrm{LC}_{50}\right)$ values extracted from the ECHA database for nickel sulfide (CAS 16812-54-7). Corresponding Klimisch scores (KS) and assigned reliability factors $\left(R_{f}=1,0.7,0.3\right.$ and 0.1 for $\mathrm{KS}=1,2,3$ and 4, respectively) are provided. Dependence of computed uncertainty scores (US) on chosen scaling factors $(y)$ is shown in columns 6-12; columns 13-14 (*) illustrate the impact of alternate translation of KS's into $R_{f}$ 's ( $R_{f}=1,0.9,0.6$ and 0.3 for $\mathrm{KS}=1,2,3$ and 4, respectively) for $y=1$ and $y=0.2$. Top-value picks with the lowest-computed uncertainty scores are outlined; top 10 values are shaded in green color to show differences due to imposed bias. Columns were sorted based on US( $\mathrm{y}=1)$ from smallest to largest value. Dataset statistics: $N=123$; arithmetic mean $=55.5 \mathrm{mg} / \mathrm{L} ;$ geometric mean $=13.7 \mathrm{mg} / \mathrm{L} ; \sigma=100.9 \mathrm{mg} / \mathrm{L} ; 95 \%$ confidence interval $=17.8 \mathrm{mg} / \mathrm{L} ;$ minimum value $=0.004168 \mathrm{mg} / \mathrm{L} ;$ maximum value $=509.4 \mathrm{mg} / \mathrm{L}$.

\begin{tabular}{|c|c|c|c|c|c|c|c|c|c|c|c|c|c|}
\hline No & KS & Rf & $\begin{array}{l}\mathrm{LC50} \\
(\mathrm{mg} / \mathrm{L})\end{array}$ & Test Species & $\begin{array}{c}\text { US } \\
(y=1)\end{array}$ & $\begin{array}{c}\text { US } \\
(y=0.8)\end{array}$ & $\begin{array}{c}\text { US } \\
(y=0.6)\end{array}$ & $\begin{array}{c}\text { US } \\
(y=0.4)\end{array}$ & $\begin{array}{c}\text { US } \\
(y=0.2)\end{array}$ & $\begin{array}{c}\text { US } \\
(y=0)\end{array}$ & $\begin{array}{c}\text { US } \\
(y=10)\end{array}$ & $\begin{array}{l}\text { US } \\
(y=1)^{*}\end{array}$ & $\begin{array}{c}\text { US } \\
(y=0.2)^{*}\end{array}$ \\
\hline 1 & 1 & 1 & 10.9 & $\begin{array}{l}\text { Oncorhynchus } \\
\text { mykiss }\end{array}$ & 0.694 & 0.666 & 0.638 & 0.608 & 0.578 & 0.547 & 1.494 & 0.686 & 0.577 \\
\hline 2 & 1 & 1 & 21.2 & $\begin{array}{l}\text { Oncorhynchus } \\
\text { mykiss }\end{array}$ & 0.710 & 0.679 & 0.648 & 0.615 & 0.582 & 0.547 & 1.565 & 0.717 & 0.584 \\
\hline 3 & 1 & 1 & 10 & $\begin{array}{l}\text { Oncorhynchus } \\
\text { mykiss }\end{array}$ & 0.730 & 0.696 & 0.661 & 0.625 & 0.587 & 0.547 & 1.649 & 0.723 & 0.585 \\
\hline 4 & 1 & 1 & 8.9 & $\begin{array}{l}\text { Oncorhynchus } \\
\text { mykiss }\end{array}$ & 0.778 & 0.736 & 0.692 & 0.646 & 0.598 & 0.547 & 1.827 & 0.771 & 0.596 \\
\hline 5 & 1 & 1 & 8.1 & $\begin{array}{l}\text { Oncorhynchus } \\
\text { mykiss }\end{array}$ & 0.815 & 0.767 & 0.716 & 0.663 & 0.607 & 0.547 & 1.951 & 0.808 & 0.605 \\
\hline 6 & 2 & 0.7 & 15.3 & $\begin{array}{l}\text { Oncorhynchus } \\
\text { mykiss }\end{array}$ & 0.913 & 0.911 & 0.909 & 0.907 & 0.906 & 0.904 & 0.989 & 0.672 & 0.656 \\
\hline 7 & 2 & 0.7 & 15.9 & $\begin{array}{l}\text { Lepomis } \\
\text { macrochirus }\end{array}$ & 0.926 & 0.922 & 0.917 & 0.913 & 0.908 & 0.904 & 1.106 & 0.689 & 0.660 \\
\hline 8 & 2 & 0.7 & 13.7 & $\begin{array}{l}\text { Oncorhynchus } \\
\text { mykiss }\end{array}$ & 0.933 & 0.928 & 0.922 & 0.916 & 0.910 & 0.904 & 1.166 & 0.683 & 0.659 \\
\hline 9 & 2 & 0.7 & 16.3 & $\begin{array}{l}\text { Oncorhynchus } \\
\text { mykiss }\end{array}$ & 0.935 & 0.928 & 0.922 & 0.916 & 0.910 & 0.904 & 1.175 & 0.700 & 0.662 \\
\hline 10 & 2 & 0.7 & 13.6 & Morone americana & 0.936 & 0.930 & 0.923 & 0.917 & 0.910 & 0.904 & 1.186 & 0.686 & 0.659 \\
\hline 11 & 2 & 0.7 & 13.57 & Puntius sophore & 0.937 & 0.930 & 0.924 & 0.917 & 0.911 & 0.904 & 1.192 & 0.687 & 0.660 \\
\hline 12 & 2 & 0.7 & 16.7 & $\begin{array}{l}\text { Oncorhynchus } \\
\text { kisutch }\end{array}$ & 0.943 & 0.935 & 0.927 & 0.920 & 0.912 & 0.904 & 1.238 & 0.710 & 0.664 \\
\hline 13 & 2 & 0.7 & 13.28 & Catla catla & 0.944 & 0.936 & 0.928 & 0.920 & 0.912 & 0.904 & 1.247 & 0.696 & 0.661 \\
\hline 14 & 2 & 0.7 & 13.12 & Danio rerio & 0.948 & 0.939 & 0.931 & 0.922 & 0.913 & 0.904 & 1.277 & 0.701 & 0.663 \\
\hline 15 & 2 & 0.7 & 17.1 & $\begin{array}{l}\text { Oncorhynchus } \\
\text { mykiss }\end{array}$ & 0.951 & 0.942 & 0.932 & 0.923 & 0.913 & 0.904 & 1.296 & 0.720 & 0.666 \\
\hline 16 & 2 & 0.7 & 13 & Anguilla rostrata & 0.951 & 0.942 & 0.933 & 0.923 & 0.914 & 0.904 & 1.299 & 0.705 & 0.663 \\
\hline 17 & 2 & 0.7 & 18 & $\begin{array}{l}\text { Oncorhynchus } \\
\text { kisutch }\end{array}$ & 0.968 & 0.955 & 0.943 & 0.930 & 0.917 & 0.904 & 1.411 & 0.741 & 0.671 \\
\hline 18 & 2 & 0.7 & 18.3 & $\begin{array}{l}\text { Lebistes } \\
\text { reticulatus }\end{array}$ & 0.973 & 0.960 & 0.946 & 0.932 & 0.918 & 0.904 & 1.445 & 0.748 & 0.672 \\
\hline 19 & 2 & 0.7 & 11.83 & Catla catla & 0.982 & 0.967 & 0.952 & 0.936 & 0.920 & 0.904 & 1.501 & 0.745 & 0.672 \\
\hline 20 & 2 & 0.7 & 18.99 & Catla catla & 0.985 & 0.970 & 0.954 & 0.937 & 0.921 & 0.904 & 1.518 & 0.763 & 0.676 \\
\hline 21 & 2 & 0.7 & 11.3 & $\begin{array}{l}\text { Lepomis } \\
\text { macrochirus }\end{array}$ & 0.997 & 0.979 & 0.961 & 0.942 & 0.923 & 0.904 & 1.586 & 0.764 & 0.676 \\
\hline 22 & 2 & 0.7 & 20.5 & Carassius auratus & 1.010 & 0.990 & 0.969 & 0.948 & 0.926 & 0.904 & 1.654 & 0.794 & 0.682 \\
\hline 23 & 2 & 0.7 & 21 & Morone saxatilis & 1.017 & 0.996 & 0.974 & 0.951 & 0.928 & 0.904 & 1.694 & 0.803 & 0.685 \\
\hline 24 & 2 & 0.7 & 21.2 & $\begin{array}{l}\text { Lepomis } \\
\text { macrochirus }\end{array}$ & 1.020 & 0.998 & 0.975 & 0.952 & 0.928 & 0.904 & 1.709 & 0.807 & 0.685 \\
\hline 25 & 2 & 0.7 & 10.4 & Cyprinus carpio & 1.023 & 1.001 & 0.977 & 0.953 & 0.929 & 0.904 & 1.723 & 0.797 & 0.683 \\
\hline 26 & 2 & 0.7 & 9.82 & Carassius auratus & 1.041 & 1.015 & 0.988 & 0.961 & 0.933 & 0.904 & 1.809 & 0.819 & 0.688 \\
\hline 27 & 2 & 0.7 & 9.56 & $\begin{array}{l}\text { Lebistes } \\
\text { reticulatus }\end{array}$ & 1.049 & 1.022 & 0.994 & 0.965 & 0.935 & 0.904 & 1.846 & 0.829 & 0.690 \\
\hline 28 & 2 & 0.7 & 24.5 & Channa punctata & 1.065 & 1.035 & 1.003 & 0.971 & 0.938 & 0.904 & 1.914 & 0.862 & 0.698 \\
\hline
\end{tabular}




\begin{tabular}{|c|c|c|c|c|c|c|c|c|c|c|c|c|c|}
\hline 29 & 2 & 0.7 & 25 & $\begin{array}{l}\text { Pimephales } \\
\text { promelas }\end{array}$ & 1.071 & 1.040 & 1.007 & 0.974 & 0.940 & 0.904 & 1.939 & 0.869 & 0.700 \\
\hline 30 & 2 & 0.7 & 25.1 & $\begin{array}{l}\text { Oncorhynchus } \\
\text { mykiss }\end{array}$ & 1.072 & 1.041 & 1.008 & 0.975 & 0.940 & 0.904 & 1.944 & 0.870 & 0.700 \\
\hline 31 & 2 & 0.7 & 8.7 & Thymallus arcticus & 1.078 & 1.045 & 1.012 & 0.977 & 0.941 & 0.904 & 1.968 & 0.864 & 0.699 \\
\hline 32 & 2 & 0.7 & 8.67 & $\begin{array}{l}\text { Lepomis } \\
\text { macrochirus }\end{array}$ & 1.079 & 1.046 & 1.012 & 0.977 & 0.941 & 0.904 & 1.972 & 0.866 & 0.699 \\
\hline 33 & 2 & 0.7 & 8.46 & Cyprinus carpio & 1.086 & 1.052 & 1.017 & 0.981 & 0.943 & 0.904 & 2.001 & 0.875 & 0.701 \\
\hline 34 & 2 & 0.7 & 26.56 & Atherinops affinis & 1.089 & 1.054 & 1.019 & 0.982 & 0.944 & 0.904 & 2.012 & 0.891 & 0.705 \\
\hline 35 & 2 & 0.7 & 27 & $\begin{array}{l}\text { Pimephales } \\
\text { promelas }\end{array}$ & 1.093 & 1.058 & 1.022 & 0.984 & 0.945 & 0.904 & 2.031 & 0.897 & 0.706 \\
\hline 36 & 2 & 0.7 & 8.2 & Thymallus arcticus & 1.095 & 1.060 & 1.023 & 0.985 & 0.945 & 0.904 & 2.037 & 0.886 & 0.704 \\
\hline 37 & 2 & 0.7 & 8 & Lepomis gibbosus & 1.102 & 1.065 & 1.027 & 0.988 & 0.947 & 0.904 & 2.065 & 0.895 & 0.706 \\
\hline 38 & 2 & 0.7 & 28 & $\begin{array}{l}\text { Pimephales } \\
\text { promelas }\end{array}$ & 1.104 & 1.067 & 1.029 & 0.989 & 0.947 & 0.904 & 2.072 & 0.909 & 0.709 \\
\hline 39 & 2 & 0.7 & 7.79 & $\begin{array}{l}\text { Oncorhynchus } \\
\text { mykiss }\end{array}$ & 1.110 & 1.072 & 1.032 & 0.991 & 0.949 & 0.904 & 2.093 & 0.904 & 0.708 \\
\hline 40 & 2 & 0.7 & 31.15 & Poecilia reticulata & 1.134 & 1.092 & 1.048 & 1.002 & 0.954 & 0.904 & 2.182 & 0.946 & 0.719 \\
\hline 41 & 2 & 0.7 & 32 & $\begin{array}{l}\text { Pimephales } \\
\text { promelas }\end{array}$ & 1.142 & 1.098 & 1.053 & 1.006 & 0.956 & 0.904 & 2.208 & 0.955 & 0.721 \\
\hline 42 & 2 & 0.7 & 32 & $\begin{array}{l}\text { Oncorhynchus } \\
\text { mykiss }\end{array}$ & 1.142 & 1.098 & 1.053 & 1.006 & 0.956 & 0.904 & 2.208 & 0.955 & 0.721 \\
\hline 43 & 2 & 0.7 & 33 & Morone saxatilis & 1.150 & 1.106 & 1.059 & 1.010 & 0.958 & 0.904 & 2.237 & 0.965 & 0.723 \\
\hline 44 & 2 & 0.7 & 33.11 & Poecilia reticulata & 1.151 & 1.106 & 1.059 & 1.010 & 0.958 & 0.904 & 2.240 & 0.966 & 0.724 \\
\hline 45 & 2 & 0.7 & 34.1 & Carassius auratus & 1.159 & 1.113 & 1.065 & 1.014 & 0.960 & 0.904 & 2.267 & 0.976 & 0.726 \\
\hline 46 & 2 & 0.7 & 34.9 & $\begin{array}{l}\text { Lebistes } \\
\text { reticulatus }\end{array}$ & 1.165 & 1.118 & 1.069 & 1.017 & 0.962 & 0.904 & 2.288 & 0.984 & 0.728 \\
\hline 47 & 2 & 0.7 & 35.5 & $\begin{array}{l}\text { Oncorhynchus } \\
\text { mykiss }\end{array}$ & 1.170 & 1.122 & 1.072 & 1.019 & 0.963 & 0.904 & 2.303 & 0.989 & 0.730 \\
\hline 48 & 2 & 0.7 & 6.2 & Morone saxatilis & 1.173 & 1.125 & 1.074 & 1.020 & 0.964 & 0.904 & 2.312 & 0.981 & 0.728 \\
\hline 49 & 2 & 0.7 & 36 & Poecilia reticulata & 1.174 & 1.125 & 1.074 & 1.021 & 0.964 & 0.904 & 2.315 & 0.994 & 0.731 \\
\hline 50 & 2 & 0.7 & 6.16 & Cyprinus carpio & 1.175 & 1.126 & 1.075 & 1.021 & 0.964 & 0.904 & 2.318 & 0.983 & 0.728 \\
\hline 51 & 2 & 0.7 & 6.1 & Cyprinus carpio & 1.177 & 1.128 & 1.077 & 1.022 & 0.965 & 0.904 & 2.326 & 0.986 & 0.729 \\
\hline 52 & 2 & 0.7 & 39.6 & $\begin{array}{l}\text { Lepomis } \\
\text { macrochirus }\end{array}$ & 1.199 & 1.147 & 1.091 & 1.033 & 0.970 & 0.904 & 2.393 & 1.024 & 0.739 \\
\hline 53 & 2 & 0.7 & 40 & $\begin{array}{l}\text { Pimephales } \\
\text { promelas }\end{array}$ & 1.202 & 1.149 & 1.093 & 1.034 & 0.971 & 0.904 & 2.401 & 1.027 & 0.740 \\
\hline 54 & 2 & 0.7 & 5.36 & $\begin{array}{l}\text { Lepomis } \\
\text { macrochirus }\end{array}$ & 1.211 & 1.157 & 1.099 & 1.038 & 0.973 & 0.904 & 2.430 & 1.028 & 0.740 \\
\hline 55 & 2 & 0.7 & 41.7 & $\begin{array}{l}\text { Lepomis } \\
\text { macrochirus }\end{array}$ & 1.213 & 1.158 & 1.100 & 1.039 & 0.974 & 0.904 & 2.433 & 1.040 & 0.743 \\
\hline 56 & 2 & 0.7 & 42.4 & $\begin{array}{l}\text { Pimephales } \\
\text { promelas }\end{array}$ & 1.217 & 1.162 & 1.103 & 1.041 & 0.975 & 0.904 & 2.446 & 1.045 & 0.744 \\
\hline 57 & 1 & 1 & 100 & Danio rerio & 1.218 & 1.115 & 1.001 & 0.871 & 0.722 & 0.547 & 2.904 & 1.223 & 0.724 \\
\hline 58 & 2 & 0.7 & 5.18 & $\begin{array}{l}\text { Lepomis } \\
\text { macrochirus }\end{array}$ & 1.220 & 1.164 & 1.105 & 1.043 & 0.976 & 0.904 & 2.456 & 1.038 & 0.742 \\
\hline 59 & 2 & 0.7 & 5.18 & $\begin{array}{l}\text { Pimephales } \\
\text { promelas }\end{array}$ & 1.220 & 1.164 & 1.105 & 1.043 & 0.976 & 0.904 & 2.456 & 1.038 & 0.742 \\
\hline 60 & 2 & 0.7 & 44.5 & $\begin{array}{l}\text { Pimephales } \\
\text { promelas }\end{array}$ & 1.229 & 1.172 & 1.111 & 1.047 & 0.978 & 0.904 & 2.482 & 1.060 & 0.748 \\
\hline 61 & 2 & 0.7 & 5 & $\begin{array}{l}\text { Pimephales } \\
\text { promelas }\end{array}$ & 1.229 & 1.172 & 1.111 & 1.047 & 0.978 & 0.904 & 2.482 & 1.049 & 0.745 \\
\hline 62 & 2 & 0.7 & 46.1 & $\begin{array}{l}\text { Fundulus } \\
\text { diaphanus }\end{array}$ & 1.238 & 1.180 & 1.117 & 1.051 & 0.980 & 0.904 & 2.507 & 1.070 & 0.751 \\
\hline 63 & 2 & 0.7 & 4.58 & $\begin{array}{l}\text { Pimephales } \\
\text { promelas }\end{array}$ & 1.251 & 1.191 & 1.126 & 1.058 & 0.984 & 0.904 & 2.544 & 1.075 & 0.753 \\
\hline 61 & $?$ &  & 1889 & $\begin{array}{l}\text { Rasbora } \\
\text { daniconius }\end{array}$ & 1252 & 1102 & 1127 & 1058 & ( & 0004 & & & 6 \\
\hline r & 2 & 0.1 & & Lebistes & 1.200 & 1.192 & 1.127 & 1.058 & 0.984 & 0.904 & 2.547 & $1.08 /$ & 0.156 \\
\hline 65 & 2 & 0.7 & 4.45 & reticulatus & 1.258 & 1.197 & 1.131 & 1.061 & 0.986 & 0.904 & 2.563 & 1.084 & 0.755 \\
\hline 66 & 2 & 0.7 & 50.12 & Poecilia reticulata & 1.259 & 1.197 & 1.132 & 1.061 & 0.986 & 0.904 & 2.565 & 1.095 & 0.758 \\
\hline 67 & 2 & 0.7 & 51 & $\begin{array}{l}\text { Oncorhynchus } \\
\text { mykiss }\end{array}$ & 1.263 & 1.201 & 1.135 & 1.063 & 0.987 & 0.904 & 2.576 & 1.100 & 0.759 \\
\hline
\end{tabular}




\begin{tabular}{|c|c|c|c|c|c|c|c|c|c|c|c|c|c|}
\hline 68 & 2 & 0.7 & 4 & $\begin{array}{l}\text { Pimephales } \\
\text { promelas }\end{array}$ & 1.284 & 1.219 & 1.149 & 1.074 & 0.992 & 0.904 & 2.632 & 1.115 & 0.764 \\
\hline 69 & 2 & 0.7 & 3.9 & Morone saxatilis & 1.290 & 1.224 & 1.153 & 1.077 & 0.994 & 0.904 & 2.648 & 1.122 & 0.766 \\
\hline 70 & 2 & 0.7 & 60.3 & $\begin{array}{l}\text { Lepomis } \\
\text { macrochirus }\end{array}$ & 1.304 & 1.235 & 1.162 & 1.083 & 0.998 & 0.904 & 2.681 & 1.147 & 0.773 \\
\hline 71 & 2 & 0.7 & 62.2 & $\begin{array}{l}\text { Lepomis } \\
\text { macrochirus }\end{array}$ & 1.311 & 1.242 & 1.167 & 1.087 & 1.000 & 0.904 & 2.699 & 1.156 & 0.775 \\
\hline 72 & 2 & 0.7 & 63 & Poecilia reticulata & 1.314 & 1.244 & 1.169 & 1.088 & 1.000 & 0.904 & 2.707 & 1.159 & 0.776 \\
\hline 73 & 2 & 0.7 & 64 & $\begin{array}{l}\text { Oreochromis } \\
\text { niloticus }\end{array}$ & 1.318 & 1.247 & 1.172 & 1.090 & 1.001 & 0.904 & 2.716 & 1.163 & 0.778 \\
\hline 74 & 2 & 0.7 & 3.4 & $\begin{array}{l}\text { Pimephales } \\
\text { promelas }\end{array}$ & 1.323 & 1.252 & 1.176 & 1.093 & 1.003 & 0.904 & 2.729 & 1.160 & 0.777 \\
\hline 75 & 2 & 0.7 & 3.18 & Cyprinus carpio & 1.338 & 1.265 & 1.186 & 1.101 & 1.007 & 0.904 & 2.766 & 1.178 & 0.782 \\
\hline 76 & 2 & 0.7 & 3.1 & $\begin{array}{l}\text { Pimephales } \\
\text { promelas }\end{array}$ & 1.344 & 1.270 & 1.190 & 1.104 & 1.009 & 0.904 & 2.780 & 1.185 & 0.784 \\
\hline 77 & 2 & 0.7 & 2.916 & $\begin{array}{l}\text { Pimephales } \\
\text { promelas }\end{array}$ & 1.358 & 1.282 & 1.200 & 1.111 & 1.013 & 0.904 & 2.812 & 1.201 & 0.789 \\
\hline 78 & 2 & 0.7 & 82.1 & $\begin{array}{l}\text { Lepomis } \\
\text { macrochirus }\end{array}$ & 1.374 & 1.296 & 1.211 & 1.119 & 1.017 & 0.904 & 2.850 & 1.229 & 0.797 \\
\hline 79 & 2 & 0.7 & 94.3 & $\begin{array}{l}\text { Oncorhynchus } \\
\text { mykiss }\end{array}$ & 1.404 & 1.322 & 1.233 & 1.135 & 1.026 & 0.904 & 2.917 & 1.263 & 0.808 \\
\hline 80 & 2 & 0.7 & 95 & Poecilia reticulata & 1.405 & 1.323 & 1.234 & 1.135 & 1.026 & 0.904 & 2.920 & 1.265 & 0.809 \\
\hline 81 & 2 & 0.7 & 2.3 & Cyprinus carpio & 1.409 & 1.327 & 1.237 & 1.137 & 1.027 & 0.904 & 2.929 & 1.261 & 0.807 \\
\hline 82 & 2 & 0.7 & 2.27 & $\begin{array}{l}\text { Pimephales } \\
\text { promelas }\end{array}$ & 1.412 & 1.329 & 1.238 & 1.139 & 1.028 & 0.904 & 2.935 & 1.264 & 0.808 \\
\hline 83 & 2 & 0.7 & 2.21 & $\begin{array}{l}\text { Pimephales } \\
\text { promelas }\end{array}$ & 1.418 & 1.334 & 1.243 & 1.142 & 1.030 & 0.904 & 2.947 & 1.271 & 0.811 \\
\hline 84 & 2 & 0.7 & 2.14 & Cyprinus carpio & 1.425 & 1.340 & 1.247 & 1.145 & 1.032 & 0.904 & 2.962 & 1.279 & 0.813 \\
\hline 85 & 2 & 0.7 & 110 & $\begin{array}{l}\text { Lepomis } \\
\text { macrochirus }\end{array}$ & 1.436 & 1.350 & 1.256 & 1.152 & 1.035 & 0.904 & 2.987 & 1.301 & 0.820 \\
\hline 86 & 2 & 0.7 & 115 & $\begin{array}{l}\text { Lepomis } \\
\text { macrochirus }\end{array}$ & 1.445 & 1.358 & 1.262 & 1.156 & 1.038 & 0.904 & 3.006 & 1.311 & 0.823 \\
\hline 87 & 2 & 0.7 & 118.3 & Chelon labrosus & 1.451 & 1.363 & 1.266 & 1.160 & 1.040 & 0.904 & 3.018 & 1.318 & 0.826 \\
\hline 88 & 2 & 0.7 & 1.68 & $\begin{array}{l}\text { Pimephales } \\
\text { promelas }\end{array}$ & 1.474 & 1.383 & 1.283 & 1.172 & 1.047 & 0.904 & 3.065 & 1.335 & 0.831 \\
\hline 89 & 2 & 0.7 & 1.64 & Cyprinus carpio & 1.479 & 1.387 & 1.286 & 1.174 & 1.048 & 0.904 & 3.075 & 1.341 & 0.833 \\
\hline 90 & 2 & 0.7 & 1.54 & Cyprinus carpio & 1.491 & 1.398 & 1.295 & 1.181 & 1.052 & 0.904 & 3.100 & 1.355 & 0.838 \\
\hline 91 & 2 & 0.7 & 150 & $\begin{array}{l}\text { Fundulus } \\
\text { heteroclitus }\end{array}$ & 1.498 & 1.404 & 1.301 & 1.185 & 1.054 & 0.904 & 3.114 & 1.371 & 0.844 \\
\hline 92 & 2 & 0.7 & 170 & $\begin{array}{l}\text { Lepomis } \\
\text { macrochirus }\end{array}$ & 1.522 & 1.425 & 1.318 & 1.198 & 1.062 & 0.904 & 3.161 & 1.399 & 0.853 \\
\hline 93 & 2 & 0.7 & 170 & $\begin{array}{l}\text { Lepomis } \\
\text { macrochirus }\end{array}$ & 1.522 & 1.425 & 1.318 & 1.198 & 1.062 & 0.904 & 3.161 & 1.399 & 0.853 \\
\hline 94 & 2 & 0.7 & 1.3 & Cyprinus carpio & 1.524 & 1.427 & 1.319 & 1.199 & 1.062 & 0.904 & 3.164 & 1.392 & 0.851 \\
\hline 95 & 2 & 0.7 & 1.3 & Cyprinus carpio & 1.524 & 1.427 & 1.319 & 1.199 & 1.062 & 0.904 & 3.164 & 1.392 & 0.851 \\
\hline 96 & 2 & 0.7 & 196 & $\begin{array}{l}\text { Oncorhynchus } \\
\text { mykiss }\end{array}$ & 1.549 & 1.449 & 1.338 & 1.213 & 1.070 & 0.904 & 3.212 & 1.429 & 0.863 \\
\hline 97 & 2 & 0.7 & 1.12 & $\begin{array}{l}\text { Pimephales } \\
\text { promelas }\end{array}$ & 1.551 & 1.451 & 1.340 & 1.215 & 1.071 & 0.904 & 3.217 & 1.424 & 0.862 \\
\hline 98 & 2 & 0.7 & 204.2 & Poecilia reticulata & 1.556 & 1.456 & 1.344 & 1.217 & 1.073 & 0.904 & 3.227 & 1.437 & 0.866 \\
\hline 99 & 2 & 0.7 & 255 & Mystus vittatus & 1.596 & 1.491 & 1.373 & 1.240 & 1.086 & 0.904 & 3.300 & 1.482 & 0.882 \\
\hline 100 & 2 & 0.7 & 0.75 & Cyprinus carpio & 1.623 & 1.514 & 1.393 & 1.255 & 1.095 & 0.904 & 3.348 & 1.505 & 0.891 \\
\hline 101 & 2 & 0.7 & 306.9 & Channa punctata & 1.628 & 1.520 & 1.398 & 1.258 & 1.097 & 0.904 & 3.358 & 1.518 & 0.896 \\
\hline 102 & 2 & 0.7 & 312 & $\begin{array}{l}\text { Oncorhynchus } \\
\text { mykiss }\end{array}$ & 1.631 & 1.522 & 1.400 & 1.260 & 1.098 & 0.904 & 3.363 & 1.521 & 0.897 \\
\hline 103 & 2 & 0.7 & 0.69 & $\begin{array}{l}\text { Pimephales } \\
\text { promelas }\end{array}$ & 1.637 & 1.527 & 1.404 & 1.263 & 1.100 & 0.904 & 3.373 & 1.521 & 0.897 \\
\hline 104 & 2 & 0.7 & 336 & Poecilia reticulata & 1.644 & 1.533 & 1.409 & 1.267 & 1.102 & 0.904 & 3.385 & 1.535 & 0.902 \\
\hline 105 & 2 & 0.7 & 350 & $\begin{array}{l}\text { Fundulus } \\
\text { heteroclitus }\end{array}$ & 1.651 & 1.539 & 1.414 & 1.271 & 1.104 & 0.904 & 3.397 & 1.543 & 0.905 \\
\hline 106 & 2 & 0.7 & 0.5899 & Danio rerio & 1.663 & 1.551 & 1.424 & 1.279 & 1.109 & 0.904 & 3.419 & 1.550 & 0.908 \\
\hline 107 & 2 & 0.7 & 400 & $\begin{array}{l}\text { Fundulus } \\
\text { heteroclitus }\end{array}$ & 1.673 & 1.559 & 1.431 & 1.284 & 1.112 & 0.904 & 3.435 & 1.567 & 0.914 \\
\hline
\end{tabular}




\begin{tabular}{|c|c|c|c|c|c|c|c|c|c|c|c|c|c|}
\hline 108 & 2 & 0.7 & 0.54 & $\begin{array}{l}\text { Pimephales } \\
\text { promelas }\end{array}$ & 1.677 & 1.563 & 1.435 & 1.287 & 1.114 & 0.904 & 3.443 & 1.566 & 0.914 \\
\hline 109 & 2 & 0.7 & 436.5 & Channa punctata & 1.687 & 1.572 & 1.442 & 1.293 & 1.117 & 0.904 & 3.459 & 1.583 & 0.920 \\
\hline 110 & 2 & 0.7 & 0.5 & $\begin{array}{l}\text { Pimephales } \\
\text { promelas }\end{array}$ & 1.690 & 1.575 & 1.444 & 1.294 & 1.118 & 0.904 & 3.465 & 1.580 & 0.919 \\
\hline 111 & 2 & 0.7 & 456 & Channa punctata & 1.694 & 1.578 & 1.447 & 1.297 & 1.119 & 0.904 & 3.471 & 1.591 & 0.923 \\
\hline 112 & 2 & 0.7 & 0.45 & $\begin{array}{l}\text { Pimephales } \\
\text { promelas }\end{array}$ & 1.707 & 1.590 & 1.457 & 1.304 & 1.124 & 0.904 & 3.493 & 1.599 & 0.926 \\
\hline 113 & 2 & 0.7 & 509.3 & Channa punctata & 1.711 & 1.594 & 1.461 & 1.307 & 1.126 & 0.904 & 3.501 & 1.610 & 0.931 \\
\hline 114 & 2 & 0.7 & 0.4 & $\begin{array}{l}\text { Pimephales } \\
\text { promelas }\end{array}$ & 1.725 & 1.606 & 1.471 & 1.315 & 1.131 & 0.904 & 3.523 & 1.619 & 0.935 \\
\hline 115 & 2 & 0.7 & 0.35 & $\begin{array}{l}\text { Pimephales } \\
\text { promelas }\end{array}$ & 1.745 & 1.625 & 1.487 & 1.328 & 1.138 & 0.904 & 3.557 & 1.642 & 0.944 \\
\hline 116 & 3 & 0.3 & 9.52 & Danio rerio & 1.817 & 1.804 & 1.791 & 1.778 & 1.765 & 1.751 & 2.268 & 1.180 & 1.084 \\
\hline 117 & 3 & 0.3 & 78 & Capoeta fusca & 1.974 & 1.933 & 1.891 & 1.846 & 1.800 & 1.751 & 3.002 & 1.466 & 1.154 \\
\hline 118 & 3 & 0.3 & 127.2 & Capoeta fusca & 2.031 & 1.981 & 1.928 & 1.873 & 1.814 & 1.751 & 3.194 & 1.560 & 1.181 \\
\hline 119 & 2 & 0.7 & 0.0042 & Liza klunzingeri & 2.259 & 2.099 & 1.907 & 1.670 & 1.359 & 0.904 & 4.298 & 2.199 & 1.206 \\
\hline 120 & 4 & 0.1 & 2.78 & Carassius auratus & 2.931 & 2.915 & 2.899 & 2.883 & 2.867 & 2.850 & 3.462 & 1.975 & 1.800 \\
\hline 121 & 4 & 0.1 & 2.06 & $\begin{array}{l}\text { Micropterus } \\
\text { salmoides }\end{array}$ & 2.945 & 2.926 & 2.908 & 2.889 & 2.869 & 2.850 & 3.540 & 2.010 & 1.809 \\
\hline 122 & 4 & 0.1 & 0.071 & Ictalurus punctatus & 3.088 & 3.045 & 2.999 & 2.952 & 2.902 & 2.850 & 4.155 & 2.341 & 1.900 \\
\hline 123 & 4 & 0.1 & 0.05 & $\begin{array}{l}\text { Oncorhynchus } \\
\text { mykiss }\end{array}$ & 3.102 & 3.056 & 3.008 & 2.958 & 2.905 & 2.850 & 4.201 & 2.370 & 1.909 \\
\hline
\end{tabular}


Table S2. Acute aquatic toxicity $\left(\mathrm{LC}_{50}\right)$ values obtained from the ECHA database for nickel sulfide (CAS 16812-54-7) as tested on Rainbow trout (Oncorhynchus mykiss). Corresponding Klimisch scores (KS) and assigned reliability factors $\left(R_{f}=1,0.7,0.3\right.$ and 0.1 for KS $=1,2,3$ and 4, respectively) are provided. Dependence of computed uncertainty scores (US) on chosen scaling factors $(y=1$ and $y=0.2)$ is shown in columns 4-5. Values with the lowest-computed uncertainty scores are outlined. Columns were sorted based on $\mathrm{US}(\mathrm{y}=1)$ from smallest to largest value. Dataset statistics: $N=20$; arithmetic mean $=45.5$ $\mathrm{mg} / \mathrm{L} ;$ geometric mean $=17.7 \mathrm{mg} / \mathrm{L} ; \sigma=76.5 \mathrm{mg} / \mathrm{L} ;$ minimum value $=0.05 \mathrm{mg} / \mathrm{L}$; maximum value $=312 \mathrm{mg} / \mathrm{L}$.

\begin{tabular}{cccccc} 
LC50 $\mathbf{~ m g / L ~}$ & Rf & KS & US $(\mathbf{y}=\mathbf{1})$ & US $(\mathbf{y}=\mathbf{0 . 2})$ \\
\cline { 4 - 5 } 21.2 & 1 & 1 & 0.404 & 0.399 \\
10.9 & 1 & 1 & 0.730 & 0.474 \\
10 & 1 & 1 & 0.766 & 0.483 \\
8.9 & 1 & 1 & 0.812 & 0.495 \\
25.1 & 0.7 & 2 & 0.817 & 0.767 \\
18 & 0.7 & 2 & 0.823 & 0.769 \\
17.1 & 0.7 & 2 & 0.843 & 0.773 \\
8.1 & 1 & 1 & 0.847 & 0.505 \\
16.7 & 0.7 & 2 & 0.851 & 0.775 \\
16.3 & 0.7 & 2 & 0.860 & 0.776 \\
15.3 & 0.7 & 2 & 0.883 & 0.782 \\
32 & 0.7 & 2 & 0.906 & 0.787 \\
13.7 & 0.7 & 2 & 0.922 & 0.790 \\
35.5 & 0.7 & 2 & 0.942 & 0.795 \\
51 & 0.7 & 2 & 1.058 & 0.823 \\
7.79 & 0.7 & 2 & 1.100 & 0.834 \\
94.3 & 0.7 & 2 & 1.228 & 0.869 \\
196 & 0.7 & 2 & 1.398 & 0.921 \\
312 & 0.7 & 2 & 1.493 & 0.952 \\
0.05 & 0.1 & 2 & 3.003 & 2.769 \\
& & & &
\end{tabular}


Table S3. Acute aquatic toxicity $\left(\mathrm{LC}_{50}\right)$ values extracted from the ECHA database for ethylbenzene (CAS 100-41-4). Corresponding Klimisch scores (KS) and assigned reliability factors $\left(R_{f}=1,0.7,0.3\right.$ and 0.1 for $\mathrm{KS}=1,2,3$ and 4, respectively) are provided. Dependence of computed uncertainty scores (US) on chosen scaling factors $(y)$ is shown in columns 5-7. Top-value picks with the lowest-computed uncertainty scores are outlined; top 10 values for each column are shaded in green color. Columns were sorted based on $\mathrm{US}(\mathrm{y}=1)$ from smallest to largest value. Dataset statistics: $N=60$; arithmetic mean $=105.8$ $\mathrm{mg} / \mathrm{L} ;$ geometric mean $=57.2 \mathrm{mg} / \mathrm{L} ; \sigma=94.4 \mathrm{mg} / \mathrm{L} ;$ minimum value $=2.4 \mathrm{mg} / \mathrm{L}$; maximum value $=360 \mathrm{mg} / \mathrm{L}$.

\begin{tabular}{|c|c|c|c|c|c|c|}
\hline No & KS & Rf & LC50 (mg/L) & US (y=1) & US $(y=0.5)$ & US $(y=0.2)$ \\
\hline 1 & 2 & 0.7 & 15 & 0.720 & 0.620 & 0.555 \\
\hline 2 & 1 & 1 & 7 & 0.796 & 0.525 & 0.318 \\
\hline 3 & 2 & 0.7 & 12.1 & 0.807 & 0.669 & 0.576 \\
\hline 4 & 1 & 1 & 6.4 & 0.831 & 0.548 & 0.329 \\
\hline 5 & 1 & 1 & 5.8 & 0.867 & 0.572 & 0.342 \\
\hline 6 & 2 & 0.7 & 9.6 & 0.893 & 0.719 & 0.598 \\
\hline 7 & 1 & 1 & 5.1 & 0.913 & 0.603 & 0.357 \\
\hline 8 & 1 & 1 & 2.4 & 1.146 & 0.768 & 0.445 \\
\hline 9 & 2 & 0.7 & 4.2 & 1.150 & 0.880 & 0.674 \\
\hline 10 & 3 & 0.3 & 26 & 1.378 & 1.367 & 1.360 \\
\hline 11 & 3 & 0.3 & 32 & 1.422 & 1.390 & 1.370 \\
\hline 12 & 3 & 0.3 & 44 & 1.487 & 1.424 & 1.384 \\
\hline 13 & 3 & 0.3 & 48.5 & 1.506 & 1.434 & 1.388 \\
\hline 14 & 3 & 0.3 & 50 & 1.512 & 1.437 & 1.389 \\
\hline 15 & 3 & 0.3 & 10 & 1.531 & 1.447 & 1.393 \\
\hline 16 & 3 & 0.3 & 70 & 1.574 & 1.471 & 1.404 \\
\hline 17 & 3 & 0.3 & 88 & 1.615 & 1.494 & 1.413 \\
\hline 18 & 3 & 0.3 & 94.4 & 1.627 & 1.500 & 1.416 \\
\hline 19 & 3 & 0.3 & 97.1 & 1.631 & 1.503 & 1.417 \\
\hline 20 & 3 & 0.3 & 4.3 & 1.678 & 1.530 & 1.429 \\
\hline 21 & 3 & 0.3 & 150 & 1.703 & 1.544 & 1.436 \\
\hline 22 & 3 & 0.3 & 169 & 1.721 & 1.555 & 1.440 \\
\hline 23 & 3 & 0.3 & 280 & 1.797 & 1.601 & 1.461 \\
\hline 24 & 3 & 0.3 & 300 & 1.807 & 1.607 & 1.464 \\
\hline 25 & 3 & 0.3 & 320 & 1.816 & 1.612 & 1.466 \\
\hline 26 & 3 & 0.3 & 360 & 1.832 & 1.622 & 1.471 \\
\hline 27 & 4 & 0.1 & 32 & 2.477 & 2.466 & 2.459 \\
\hline 28 & 4 & 0.1 & 14 & 2.493 & 2.474 & 2.462 \\
\hline 29 & 4 & 0.1 & 14 & 2.493 & 2.474 & 2.462 \\
\hline 30 & 4 & 0.1 & 14 & 2.493 & 2.474 & 2.462 \\
\hline 31 & 4 & 0.1 & 12.1 & 2.503 & 2.479 & 2.464 \\
\hline
\end{tabular}




\begin{tabular}{|c|c|c|c|c|c|c|}
\hline 32 & 4 & 0.1 & 10.5 & 2.513 & 2.484 & 2.466 \\
\hline 33 & 4 & 0.1 & 56 & 2.517 & 2.486 & 2.467 \\
\hline 34 & 4 & 0.1 & 80 & 2.542 & 2.499 & 2.473 \\
\hline 35 & 4 & 0.1 & 80 & 2.542 & 2.499 & 2.473 \\
\hline 36 & 4 & 0.1 & 84 & 2.545 & 2.501 & 2.473 \\
\hline 37 & 4 & 0.1 & 86 & 2.547 & 2.502 & 2.474 \\
\hline 38 & 4 & 0.1 & 88 & 2.548 & 2.503 & 2.474 \\
\hline 39 & 4 & 0.1 & 88 & 2.548 & 2.503 & 2.474 \\
\hline 40 & 4 & 0.1 & 90 & 2.550 & 2.503 & 2.474 \\
\hline 41 & 4 & 0.1 & 94.44 & 2.553 & 2.505 & 2.475 \\
\hline 42 & 4 & 0.1 & 97.1 & 2.555 & 2.506 & 2.475 \\
\hline 43 & 4 & 0.1 & 97.1 & 2.555 & 2.506 & 2.475 \\
\hline 44 & 4 & 0.1 & 100 & 2.557 & 2.507 & 2.476 \\
\hline 45 & 4 & 0.1 & 134 & 2.577 & 2.517 & 2.480 \\
\hline 46 & 4 & 0.1 & 134 & 2.577 & 2.517 & 2.480 \\
\hline 47 & 4 & 0.1 & 135 & 2.577 & 2.518 & 2.480 \\
\hline 48 & 4 & 0.1 & 135 & 2.577 & 2.518 & 2.480 \\
\hline 49 & 4 & 0.1 & 135 & 2.577 & 2.518 & 2.480 \\
\hline 50 & 4 & 0.1 & 135 & 2.577 & 2.518 & 2.480 \\
\hline 51 & 4 & 0.1 & 140 & 2.579 & 2.519 & 2.481 \\
\hline 52 & 4 & 0.1 & 160 & 2.588 & 2.524 & 2.483 \\
\hline 53 & 4 & 0.1 & 198 & 2.602 & 2.531 & 2.486 \\
\hline 54 & 4 & 0.1 & 210 & 2.606 & 2.533 & 2.487 \\
\hline 55 & 4 & 0.1 & 210 & 2.606 & 2.533 & 2.487 \\
\hline 56 & 4 & 0.1 & 210 & 2.606 & 2.533 & 2.487 \\
\hline 57 & 4 & 0.1 & 220 & 2.609 & 2.535 & 2.487 \\
\hline 58 & 4 & 0.1 & 275 & 2.623 & 2.542 & 2.491 \\
\hline 59 & 4 & 0.1 & 285 & 2.625 & 2.543 & 2.491 \\
\hline 60 & 4 & 0.1 & 285 & 2.625 & 2.543 & 2.491 \\
\hline
\end{tabular}


Table S4. A questionnaire for the MUST parametrization. Instructions to users: For the following cases, 1-39, select the order of values corresponding to high, medium and low toxicological concern as you would in the context of your decision-making process. The first value should be your top pick; the last value should be the study you are least likely to rely on. Color coding assigns relative reliability of the study - rows 1-27 (part A): green = high, yellow = medium, red = low; rows 28-39 (part B): 1 (least-reliable) to 10 (mostreliable). Input your selection in the column marked "User selection". Example, Case 1: 2, 1,3 .

\begin{tabular}{|c|c|c|c|c|c|}
\hline A & $\begin{array}{l}\text { High Tox Value } \\
\text { Study } 1\end{array}$ & $\begin{array}{l}\text { Med Tox Value } \\
\text { Study } 2\end{array}$ & $\begin{array}{l}\text { Low Tox Value } \\
\text { Study } 3\end{array}$ & User selection & \\
\hline 1 & & & & & High Reliability \\
\hline 2 & & & & & Medium Reliability \\
\hline 3 & & & & & Low Reliability \\
\hline 4 & & & & & \\
\hline 5 & & & & & \\
\hline 6 & & & & & \\
\hline 7 & & & & & \\
\hline 8 & & & & & \\
\hline 9 & & & & & \\
\hline 10 & & & & & \\
\hline 11 & & & & & \\
\hline 12 & & & & & \\
\hline 13 & & & & & \\
\hline 14 & & & & & \\
\hline 15 & & & & & \\
\hline 16 & & & & & \\
\hline 17 & & & & & \\
\hline 18 & & & & & \\
\hline 19 & & & & & \\
\hline 20 & & & & & \\
\hline 21 & & & & & \\
\hline 22 & & & & & \\
\hline 23 & & & & & \\
\hline 24 & & & & & \\
\hline 25 & & & & & \\
\hline 26 & & & & & \\
\hline 27 & & & & & \\
\hline B & & & & & \\
\hline 28 & 10 & 9 & 9 & & \\
\hline 29 & 10 & 9 & 8 & & \\
\hline 30 & 10 & 8 & 7 & & \\
\hline 31 & 10 & 8 & 6 & & \\
\hline 32 & 10 & 7 & 5 & & \\
\hline 33 & 10 & 7 & 4 & & \\
\hline 34 & 9 & 9 & 10 & & \\
\hline 35 & 8 & 9 & 10 & & \\
\hline 36 & 7 & 8 & 10 & & \\
\hline 37 & 6 & 8 & 10 & & \\
\hline 38 & 5 & 7 & 10 & & \\
\hline 39 & 4 & 7 & 10 & & \\
\hline
\end{tabular}

